Claremont Colleges

Scholarship@ Claremont

All HMC Faculty Publications and Research

HMC Faculty Scholarship

4-1-2000

\title{
Counting on Continued Fractions
}

Arthur T. Benjamin

Harvey Mudd College

Francis E. Su

Harvey Mudd College

Jennifer J. Quinn

Occidental College

\section{Recommended Citation}

Benjamin, Arthur T., Francis E. Su and Jennifer J. Quinn. "Counting on Continued Fractions." Mathematics Magazine, Vol 73, No. 2, pp. 98-104, April, 2000.

This Article is brought to you for free and open access by the HMC Faculty Scholarship at Scholarship @ Claremont. It has been accepted for inclusion in All HMC Faculty Publications and Research by an authorized administrator of Scholarship @ Claremont. For more information, please contact scholarship@cuc.claremont.edu. 


\title{
Counting on Continued Fractions
}

\author{
ARTHUR T. BENJAMIN \\ FRANCIS EDWARD SU \\ Harvey Mudd College \\ Claremont, CA 91711 \\ JENNIFER J. QUINN \\ Occidental College \\ Los Angeles, CA 90041
}

\section{Introduction}

You might be surprised to learn that the finite continued fraction

$$
3+\frac{1}{7+\frac{1}{15+\frac{1}{1+\frac{1}{292}}}} \text { and its reversal } 292+\frac{1}{1+\frac{1}{15+\frac{1}{7+\frac{1}{3}}}}
$$

have the same numerator. These fractions simplify to $\frac{103993}{33102}$ and $\frac{103993}{355}$ respectively. In this paper, we provide a combinatorial interpretation for the numerators and denominators of continued fractions which makes this reversal phenomenon easy to see. Through the use of counting arguments, we illustrate how this and other important identities involving continued fractions can be easily visualized, derived, and remembered.

We begin by defining some basic terminology. Given an infinite sequence of integers $a_{0} \geq 0, a_{1} \geq 1, a_{2} \geq 1, \ldots$, let $\left[a_{0}, a_{1}, \ldots, a_{n}\right]$ denote the finite continued fraction

$$
a_{0}+\frac{1}{a_{1}+\frac{1}{a_{2}+\frac{1}{\ddots+\frac{1}{a_{n}}}}}
$$

The infinite continued fraction $\left[a_{0}, a_{1}, a_{2}, \ldots\right]$ is the limit of $\left[a_{0}, a_{1}, \ldots, a_{n}\right]$ as $n \rightarrow \infty$. This limit always exists and is some irrational number $\alpha$ [3]. The rational number $r_{n}:=\left[a_{0}, a_{1}, \ldots, a_{n}\right]$ is a fraction $p_{n} / q_{n}$ in lowest terms, called the $n$-th convergent of $\alpha$. It is well-known that $p_{n}$ and $q_{n}$ satisfy the recurrences

$$
\begin{aligned}
& p_{n}=a_{n} p_{n-1}+p_{n-2} \\
& q_{n}=a_{n} q_{n-1}+q_{n-2}
\end{aligned}
$$

for $n \geq 2$, with initial conditions $p_{0}=a_{0}, p_{1}=a_{1} a_{0}+1, q_{0}=1, q_{1}=a_{1}$.

Now let's do some combinatorics. For a given continued fraction $\left[a_{0}, a_{1}, a_{2}, \ldots\right]$, consider the following tiling problem. Let $P_{n}$ count the number of ways to tile a $1 \times(n+1)$ board with dominoes and stackable square tiles. All cells (numbered $0,1, \ldots, n)$ must be covered by a tile. Nothing can be stacked on top of a domino, but cell number $i$ may be covered by a stack of as many as $a_{i}$ square tiles, $i=0, \ldots, n$. 
Figure 1 shows an empty board with the height conditions $a_{0}, a_{1}, \ldots, a_{n}$ indicated. Figure 2 gives an example of a valid tiling for a $1 \times 12$ board with height conditions $5,10,3,1,4,8,2,7,7,4,2,3$.

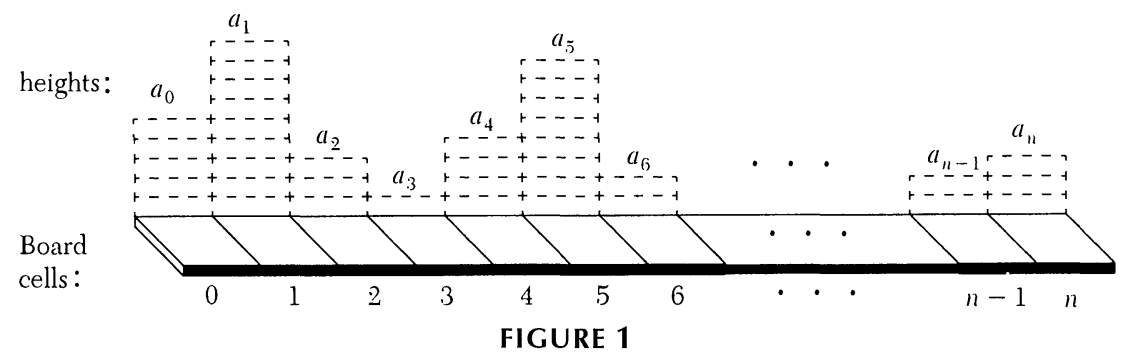

An empty $1 \times(n+1)$ board.

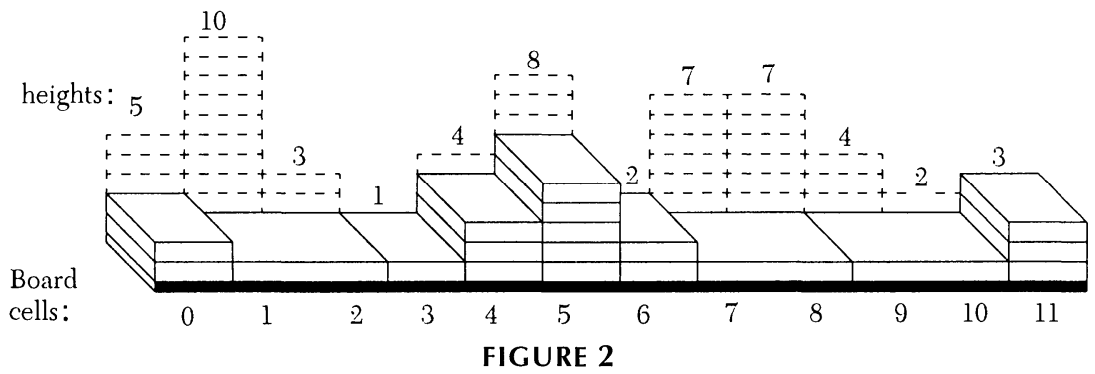

A tiling satisfying the height conditions $5,10,3,1,4,8,2,7,7,4,2,3$.

For $n \geq 2$, we show

$$
P_{n}=a_{n} P_{n-1}+P_{n-2} .
$$

This follows from the observation that a tiling either ends with a stack of square tiles or a single domino. In the first case, there are $a_{n}$ choices for the stack size and $P_{n-1}$ ways to tile cells 0 through $n-1$. In the second case, there is only one choice for the last domino, and there are $P_{n-2}$ ways to tile cells 0 through $n-2$. Using Figure 3 one can check that $P_{0}=a_{0}$ and $P_{1}=a_{0} a_{1}+1$. Since $P_{n}$ and $p_{n}$ satisfy the same recurrence and initial conditions, we have $P_{n}=p_{n}$.
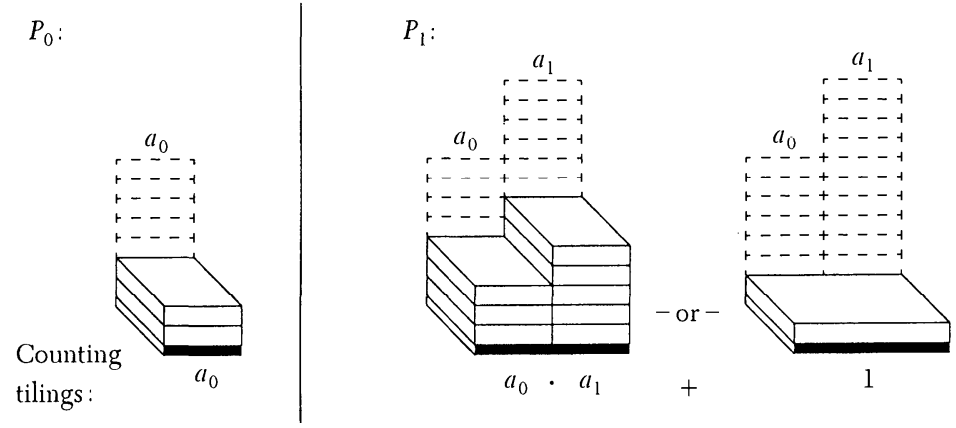

FIGURE 3

Verifying the initial conditions for the recurrence relation $P_{n}=a_{n} P_{n-1}+P_{n-2}$. 
Removing cell 0 from the previous board, let $Q_{n}$ count the number of ways to tile the $1 \times n$ board with dominoes and stackable square tiles, where the $i$ th cell may be covered by a stack of as many as $a_{i}$ square tiles, $i=1, \ldots, n$. By the same reasoning as before, (and letting $Q_{0}=1$ denote the "empty" tiling) we see that $Q_{n}=q_{n}$.

To illustrate, consider the continued fraction representation for $\pi$, which begins $[3,7,15,1,292, \ldots]$. See Figure 4 . If we count the number of ways to tile cells 0,1 , and 2 , we get $p_{2}=333$. Counting the number of ways to tile only cells 1 and 2 easily gives us $q_{2}=106$. This produces the $\pi$ approximation $r_{2}=333 / 106$. The reader should verify that tiling cells 0 through 3 produces $r_{3}=355 / 113$.

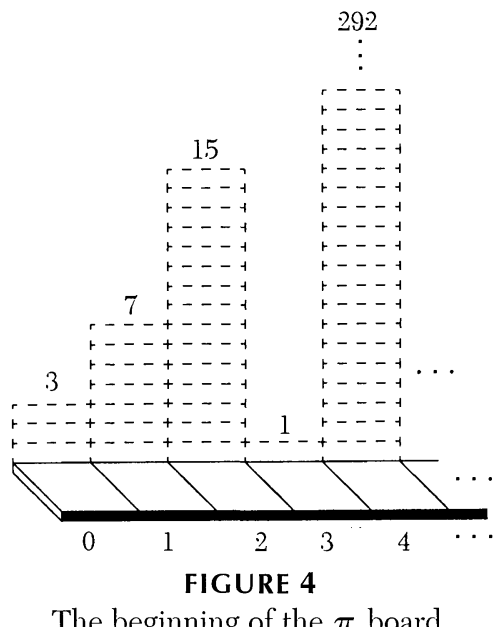

The beginning of the $\pi$ board.

When $a_{i}=1$ for all $i \geq 0$, it is well-known that the $n$th convergent $p_{n} / q_{n}$ is the ratio of two consecutive Fibonacci numbers. Specifically, if we define $f_{0}=1, f_{1}=1$, and for $n \geq 2, f_{n}=f_{n-1}+f_{n-2}$, then $p_{n}=f_{n+1}$ and $q_{n}=f_{n}$. You may recall that the Fibonacci number $f_{n}$ counts the number of ways to tile a $1 \times n$ board with $1 \times 1$ squares and $1 \times 2$ dominoes. So the continued fraction tiling problem generalizes the tiling interpretation of Fibonacci numbers [1, 2].

\section{Identities}

Armed with our tiling interpretation, many well-known continued fraction identities can be explained combinatorially. We begin with the reversal identity.

THeorem 1. If $\left[a_{0}, a_{1}, \ldots, a_{n-1}, a_{n}\right]=p_{n} / q_{n}$, then $\left[a_{n}, a_{n-1}, \ldots, a_{1}, a_{0}\right]=$ $p_{n} / p_{n-1}$.

Proof. Although one can easily prove this by induction, the theorem is nearly obvious when viewed combinatorially. To understand the common numerator, we see that the number of ways to tile the board with height conditions $a_{n}, a_{n-1}, \ldots, a_{1}, a_{0}$ is the same as the number of ways to tile the board with height conditions $a_{0}, a_{1}, \ldots, a_{n-1}, a_{n}$. The denominator of $\left[a_{n}, a_{n-1}, \ldots, a_{1}, a_{0}\right]$ is the number of ways to tile the board with height conditions $\left[a_{n-1}, \ldots, a_{1}, a_{0}\right]$, which by reversal is $p_{n-1}$.

The next few identities are useful for measuring the rate of convergence of convergents. 
THEOREM 2. The difference between consecutive convergents of $\left[a_{0}, a_{1}, a_{2}, \ldots\right]$ is: $r_{n}-r_{n-1}=(-1)^{n-1} / q_{n} q_{n-1}$. Equivalently, after multiplying both sides by $q_{n} q_{n-1}$, we have

$$
p_{n} q_{n-1}-p_{n-1} q_{n}=(-1)^{n-1}
$$

Proof. Given height conditions $a_{0}, a_{1}, \ldots, a_{n}$, let $\mathscr{P}_{n}$ denote the set of all tilings on cells $0, \ldots, n$ and let $\mathscr{Q}_{n}$ denote the set of all tilings on cells $1, \ldots, n$. Note that these sets have sizes $\left|\mathscr{P}_{n}\right|=p_{n}$ and $\left|\mathscr{Q}_{n}\right|=q_{n}$.

We will construct an almost one-to-one correspondence between the sets $\mathscr{P}_{n} \times \mathscr{Q}_{n-1}$ and $\mathscr{P}_{n-1} \times \mathscr{Q}_{n}$. Consider $(S, T) \in \mathscr{P}_{n} \times \mathscr{Q}_{n-1}$. For $i \geq 1$, we say $(S, T)$ has a fault at cell $i$ if both $S$ and $T$ have tiles that end at $i$. We say $(S, T)$ has a fault at cell 0 if $S$ has a square at cell 0 . For instance, in Figure 5, there are faults at cells $0,3,5$, and 6 .

If $(S, T)$ has a fault, construct $\left(S^{\prime}, T^{\prime}\right)$ by swapping the "tails" of $S$ and $T$ after the rightmost fault. See Figures 5 and 6 . Note that $\left(S^{\prime}, T^{\prime}\right) \in \mathscr{P}_{n-1} \times \mathscr{Q}_{n}$. Since $\left(S^{\prime}, T^{\prime}\right)$ has the same rightmost fault as $(S, T)$, this procedure is completely reversible.

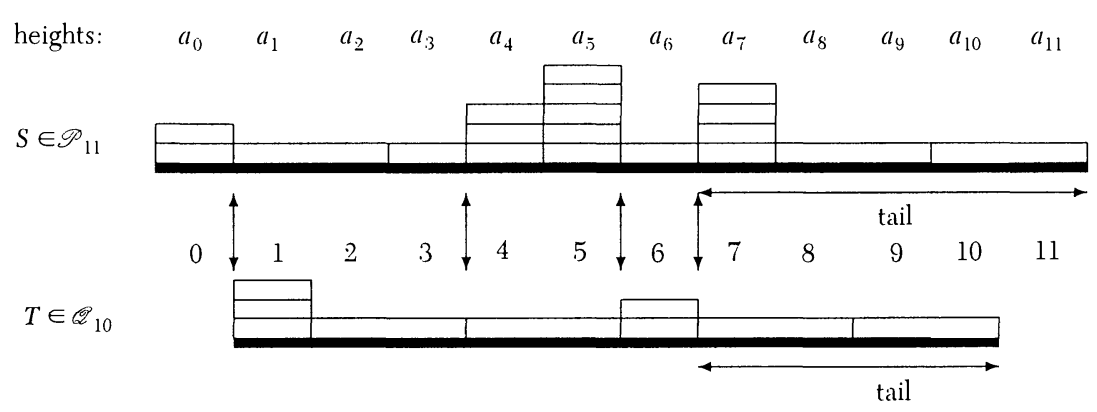

FIGURE 5

A pair of tilings with faults and tails indicated.

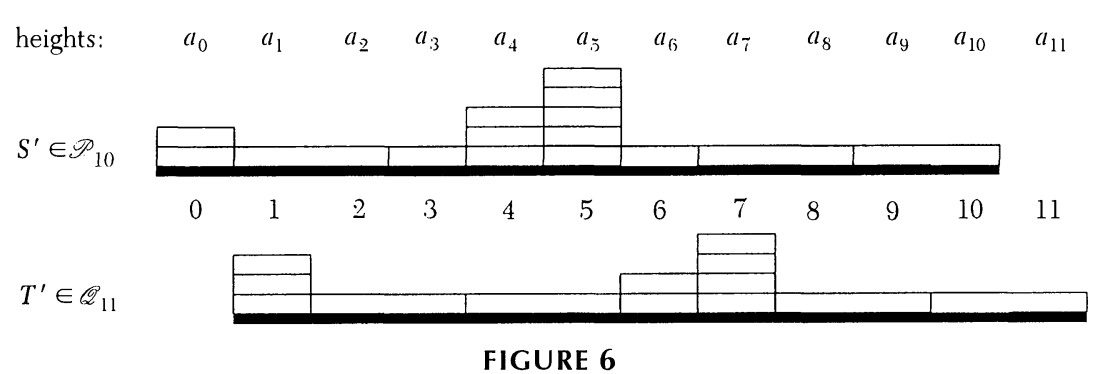

Result of swapping tails in Figure 5.

Notice when either $S$ or $T$ contains a square, $(S, T)$ must have a fault. Thus the only fault-free pairs occur when $S$ and $T$ consist of all dominoes in staggered formation as illustrated in Figure 7. When $n$ is odd (i.e., $S$ and $T$ both cover an even number of cells), there is precisely one fault-free element of $\mathscr{P}_{n} \times \mathscr{Q}_{n-1}$ and no fault-free elements of $\mathscr{P}_{n-1} \times \mathscr{Q}_{n}$. Therefore when $n$ is odd, we have $\left|\mathscr{P}_{n} \times \mathscr{Q}_{n-1}\right|$ $-\left|\mathscr{P}_{n-1} \times \mathscr{Q}_{n}\right|=1$. 


\begin{tabular}{|c|c|c|c|c|c|c|c|c|c|c|}
\hline$a_{0}$ & $a_{1}$ & $a_{2}$ & $a_{3}$ & $a_{4}$ & $a_{\tilde{3}}$ & $a_{6}$ & $a_{7}$ & $a_{8}$ & $a_{y}$ & $a_{10}$ \\
\hline 0 & 1 & 2 & 3 & 4 & 5 & 6 & 7 & 8 & 9 & 10 \\
\hline
\end{tabular}

FIGURE 7

The fault-free pair consists of staggered dominoes.

Similarly when $n$ is even, there are no fault-free elements of $\mathscr{P}_{n} \times \mathscr{Q}_{n-1}$ and exactly one fault-free element of $\mathscr{P}_{n-1} \times \mathscr{Q}_{n}$. Hence when $n$ is even, $\left|\mathscr{P}_{n} \times \mathscr{Q}_{n-1}\right|$ $-\left|\mathscr{P}_{n-1} \times \mathscr{Q}_{n}\right|=-1$. Treating the odd and even cases together, we obtain

$$
p_{n} q_{n-1}-p_{n-1} q_{n}=(-1)^{n-1} .
$$

The next identity shows that the even convergents are increasing, while the odd convergents are decreasing.

THEOREM 3. $r_{n}-r_{n-2}=(-1)^{n} a_{n} / q_{n} q_{n-2}$. Equivalently, after multiplying both sides by $q_{n} q_{n-2}$, we have

$$
p_{n} q_{n-2}-p_{n-2} q_{n}=(-1)^{n} a_{n} .
$$

Proof. As in the last proof, we use tail swapping after the last fault to create a one-to-one correspondence between the "faulty" elements of $\mathscr{P}_{n} \times \mathscr{Q}_{n-2}$ and $\mathscr{P}_{n-2}$ $\times \mathscr{Q}_{n}$. The proof is essentially given in Figures 8, 9, and 10 .

The only unmatched elements are those that are fault-free. When $n$ is odd, there are no fault-free elements of $\mathscr{P}_{n} \times \mathscr{Q}_{n-2}$, but there are precisely $a_{n}$ fault-free

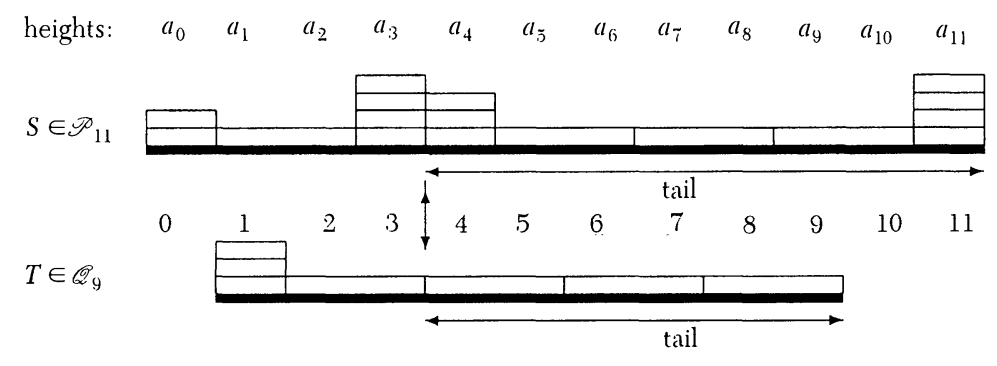

FIGURE 8

An element of $\mathscr{P}_{11} \mathscr{Q}_{9}$ with rightmost fault indicated.

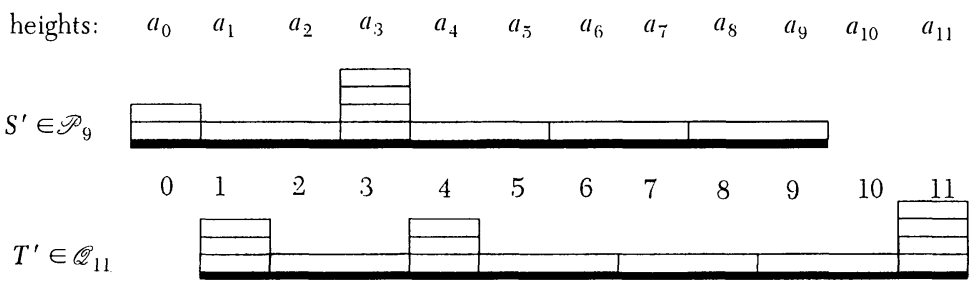

FIGURE 9

The result of swapping tails in Figure 8. 


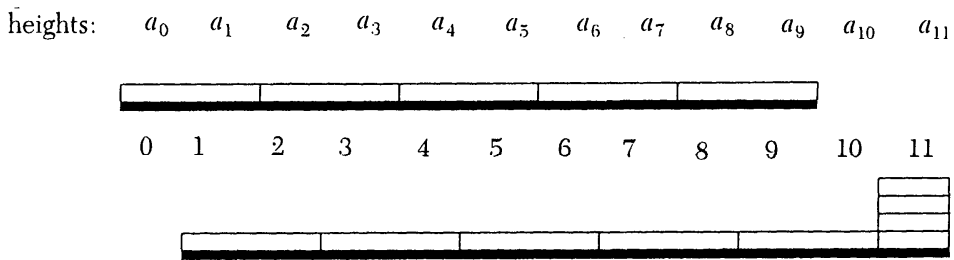

FIGURE 10

Problem pairings are fault-free.

elements of $\mathscr{P}_{n-2} \times \mathscr{Q}_{n}$, consisting of a stack of squares on the $n$th cell, and dominoes everywhere else (FIGURE 10). Likewise when $n$ is even, there are no fault-free elements of $\mathscr{P}_{n-2} \times \mathscr{Q}_{n}$, but there are $a_{n}$ fault-free elements of $\mathscr{P}_{n} \times \mathscr{Q}_{n-2}$, consisting of a stack of squares on the $n$th cell, and dominoes everywhere else. Thus we have established $\left|\mathscr{P}_{n} \times \mathscr{Q}_{n-2}\right|-\left|\mathscr{P}_{n-2} \times \mathscr{Q}_{n}\right|=(-1)^{n} a_{n}$, as desired.

Using the combinatorially clear fact that $q_{n} \rightarrow \infty$ as $n \rightarrow \infty$, the last two identities demonstrate that $\left(r_{0}, r_{1}\right),\left(r_{2}, r_{3}\right),\left(r_{4}, r_{5}\right), \ldots$ is a sequence of nested intervals whose lengths are going to zcro. Hence, $\lim _{n \rightarrow \infty} r_{n}$ exists.

\section{Extensions}

Next we examine the quantity $K(i, j)$, for $i \leq j$, that counts the number of tilings of the sub-board with cells $i, i+1, \ldots, j$ with height conditions $a_{i}, a_{i+1}, \ldots, a_{j}$. For convenience we define $K(i, i-1)=1$. We see that $K(i, j)$ is the numerator of the continued fraction $\left[a_{i}, a_{i+1}, \ldots, a_{j}\right]$ and the denominator of the continued fraction $\left[a_{i-1}, a_{i}, \ldots, a_{j}\right]$. Thus the $K(i, j)$ are identical to the classical continuants of Euler [4].

The following theorem, due to Euler, can also be proved by the same tail-swapping technique.

THEOREM 4. For $i<m<j<n$,

$$
K(i, j) K(m, n)-K(i, n) K(m, j)=(-1)^{j-m} K(i, m-2) K(j+2, n) .
$$

This result follows by considering tilings of sub-boards $S$ from cells $i$ to $j$ and $T$ from $m$ to $n$. Every faulty pair $(S, T)$ corresponds to another faulty pair $\left(S^{\prime}, T^{\prime}\right)$ obtained by swapping the tails after the last fault. The term on the right side of Theorems 4 counts the number of fault-free tilings that only occur when the overlapping regions (of $S$ and $T$, or of $S^{\prime}$ and $T^{\prime}$, depending on the parity of $j-m$ ) consist entirely of dominoes in staggered formation. See FIgures 11 and 12. Setting $i=0$ and $m=1$, Theorem 4 generalizes Theorems 2 and 3 by allowing us to compare arbitrary convergents $r_{j}$ and $r_{n}$.

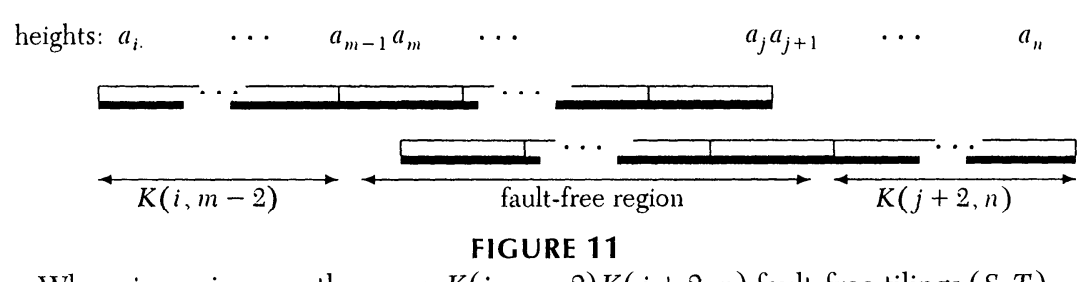

When $j-m$ is even, there are $K(i, m-2) K(j+2, n)$ fault-free tilings $(S, T)$. 


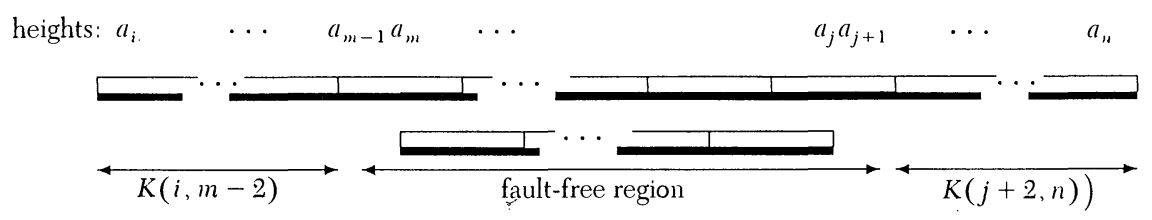

FIGURE 12

When $j-m$ is odd, there are $K(i, m-2) K(j+2, n)$ fault-free tilings $\left(S^{\prime}, T^{\prime}\right)$.

Finally, we generalize in a different direction. Suppose we allow dominoes to be stacked as well as squares. Specifically, suppose we impose height conditions $b_{1}, b_{2}, \ldots$ so that we may stack as many as $b_{i}$ dominoes on cells $i-1$ and $i$. We let $\hat{P}_{n}$ count the number of ways to tile the board with cells $0,1, \ldots, n$ and height conditions $a_{0}, \ldots, a_{n}$ and $b_{1}, \ldots, b_{n}$ for the squares and dominoes respectively. We let $\hat{Q}_{n}$ count the same problem with cell 0 removed. As before, we see that $\hat{P}_{n}$ and $\hat{Q}_{n}$ satisfy

$$
\begin{aligned}
& \hat{P}_{n}=a_{n} \hat{P}_{n-1}+b_{n} \hat{P}_{n-2} \\
& \hat{Q}_{n}=a_{n} \hat{Q}_{n-1}+b_{n} \hat{Q}_{n-2}
\end{aligned}
$$

for $n \geq 2$, with initial conditions $\hat{P}_{0}=a_{0}, \hat{P}_{1}=a_{1} a_{0}+b_{1}, \hat{Q}_{0}=1, \hat{Q}_{1}=a_{1}$. But these are precisely the conditions that define the convergents of the expansion

$$
a_{0}+\frac{b_{1}}{a_{1}+\frac{b_{2}}{a_{2}+\frac{b_{3}}{\ddots+\frac{b_{n}}{a_{n}+} \cdot}}}
$$

In other words, when the above continued fraction is truncated at the $b_{n} / a_{n}$ term, it simplifies to the rational number $\hat{P}_{n} / \hat{Q}_{n}$. All of the preceding theorems have generalizations along these lines with similar combinatorial interpretations. We invite the reader to continue these investigations.

Acknowledgment. We thank Chris Hanusa for asking an inspiring question.

\section{REFERENCES}

1. A. T. Benjamin and J. J. Quinn, Recounting Fibonacci and Lucas identities, College Math. J. 30 (1999), 359-366.

2. R. C. Brigham, R. M. Caron, P. Z. Chinn, and R. P. Grimaldi, A tiling scheme for the Fibonacci numbers, J. Recreational Math., Vol. 28, No. 1 (1996-97), 10-16.

3. I. Niven, H. S. Zuckerman, and H. L. Montgomery, An Introduction to the Theory of Numbers, John Wiley and Sons, Inc., New York, NY, 1991.

4. O. Perron, Die Lehre von den Kettenbriichen, Chelsea Publishing Co., New York, NY, 1929.

5. S. Vajda, Fibonacci and Lucas Numbers, and the Golden Section, John Wiley and Sons, New York, NY, 1989. 